\title{
THE RELIABILITY OF THE DISSOCIATION-VALUES DETERMINED BY ELECTRICAL CONDUCTIVITY MEASUREMENTS.'
}

By Arthur A. Noyes.

Received May 25, 3898.

I. VAN LAAR'S CORRECTION OF THE HEAT OF SOLUTION FORMULA.

THERE has recently appeared ${ }^{2}$ an article by van Laar in which he claims with the greatest positiveness that the dissociation-values calculated from the electrical conductivity are incorrect. In order to sustain this view, he brings forward certain "proofs" and offers an bypothesis concerning the cause of the supposed unreliability of the conductivity method. The matter is of so great importance and the view of van Laar is, in my opinion, so completely unjustifiable, that I feel compelled to reply to his arguments, and to present the existing proofs of the contrary idea.

As an evidence of his view, van Laar attempted to show that the heats of solution, calculated thermodynamically from the changes in solubility caused by variations of temperature, agreed much better with the experimental values when the theoretical (Ostwald) dilution-law was used in the calculation, than when the empirical (Rudolphi-van't Hoff) law derived from conductivity values was employed. Unfortunately, however, van Laar has not, it seems to me, derived correctly the relation between the change in solubility and heat of solution in the case of dissociated substances. It is apparent that his equation in the derivation of which the empirical dilution-law was assumed is incorrect, since this furnishes an evidently false result in the limiting case of complete dissociation. The equation to which I refer is as follows :

$$
\frac{L}{R T^{2}}=\frac{3}{3-a} \frac{d \log s}{d T}, \ldots \ldots \ldots \ldots \text { (I) }
$$

in which $L$ expresses the heat of solution; $R$, the gas constant ; $T$, the absolute temperature; $a$, the degree of dissociation; and $s$, the solubility. When $a=\mathrm{I}$, this equation becomes

1 Presented at a meeting of the North Eastern Section of the Society.

2 Ztschr. phys. Chem., ar, 79. 


$$
\frac{L}{R T^{2}}=-\frac{3}{2} \frac{d \log s}{d T}, \ldots \ldots \ldots \ldots(2)
$$

For complete dissociation, however, this equation must evidently be quite independent of any dilution-law whatever; for no change in the degree of dissociation takes place in this case. Also, since in the case of a wholly dissociated di-ionic salt the equilibrium constant $K$, which occurs in the general van't Hoff equation,

$$
\frac{d \log K}{d T}=\frac{Q}{R T^{2}}
$$

is equal to $s^{2}$, the product of the concentrations of the ions, it follows that

$$
\frac{L}{R T^{2}}=\frac{2 d \log s}{d T}, \ldots \ldots \ldots \ldots \ldots(3)
$$

an equation which has already ${ }^{1}$ been employed and confirmed. Even the equation derived by van Laar under the assumption of the theoretical dilution-law,

$$
\frac{L}{R T^{2}}=\frac{2}{2-a} \frac{d \log s}{d T}, \ldots \ldots \ldots \ldots(4)
$$

becomes simplified to equation (3) when $a=\mathrm{I}$. His two equations (2) and (4) therefore lead to different results in the case where the influence of the dilution-law must disappear. Moreover, even the last equation of van Laar cannot be correct in general, although it fulfils the conditions of the two limiting cases; for it contains no term which represents the change of the dissociation with the temperature. This is, however, obviously necessary ; for the heat of solution of a partially dissociated substance may be regarded as the sum of two quantities, one of which depends on the heat of solution of the undissociated substance, and the other on its heat of dissociation. This latter amount of heat is, however, determined thermodynamically by the temperature-change of the dissociation-constant, while the former is independent of it. Accordingly the sum, the actual heat of solution, must be a function of the temperature-change of the dissociation-constant.

1 Nernst: Theoretische Chemie, ${ }^{2} 16$. 
The original equation of van't $\mathrm{Hoff}^{1}$

$$
\frac{L}{R T^{2}}=\frac{i d \log i s}{d T}=(\mathrm{I}+a) \frac{d \log s}{d T}+\frac{d a}{d T} \cdots
$$

satisfies this theoretical requirement. That of van Laar is an attempted improvement on this. According to him the assumption of van't Hoff ${ }^{2}$ and Rudolphi ${ }^{3}$ that $L=W+a Q$, where $L$ is the actual (total) heat of solution, $W$, the heat of solution of the undissociated substance, and $Q$, its heat of dissociation, is not correct; but since in the solution of the quantity $d s$ there takes place, besides its own change in dissuciation, a change in the degree of dissociation of the total quantity $s$ of the substance present, by an amount $\frac{d a}{d s} d s$, it follows that $L d s=W d s+$ $\left(a+s \frac{d a}{d s}\right) Q d s$, in which the quantity $\frac{d a}{d s}$ is to be obtained from the dilution-law. For the heat of solution, $L$, of a grammolecule, it follows, therefore, according to van Laar, that

$$
L=W\left(a+s \frac{d a}{d s}\right) Q .
$$

This "correction" seems to me, however, to be wholly erroneous and to arise from a misunderstanding of the significance of the heat of solution. The molecular heat of solution is, in a thermodynamical sense, the heat which is absorbed by the dissolving of one molecule of the substance in a solution of unchangeable concentration; namely, that corresponding to the equilibrium. Even differential changes of concentration must not occur if the thermodynamical relations are to hold strictly; and if they actually occur, as van Laar assumes, the heat of solution then obtained will differ from the thermodynamical heat by an amount corresponding to the influence of those changes in concentration; that is to say, the dissolving must take place with a simultaneous increase in the volume of the solution by the addition of the solvent, in such a way that the concentration remains constant, just as in the reversible evaporation of a liquid the volume of the vapor must increase continuously.

1 Ztschr.phys. Chem., I7, $147 ; i=(I+a)$ for a di-ionic electrolyte.

2 Ibid., 17, 547.

8 Ibid., 17, 299. 
By these considerations it seems to me that the falsity of van Laar's attempted correction of van't Hoff's equation is made evident. The matter is, however, so important, as well from a general point of view as from the standpoint of this discussion, that it seems proper to present a direct derivation of the van't Hoff equation by means of a cyclical process, and especially so since van't Hoff himself, according to the assertion of van Laar, ${ }^{1}$ has acknowledged the validity of the latter's proposed correction.

2. DERIVATION OF THE RELATION BETWEEN THE HEAT OF SOLUTION OF DISSOCIATED SUBSTANCES AND THE CHANGE

IN THEIR SOLUBILITY WITH THE TEMPERATURE.

In order to derive this relation we will consider a cyclical process to be carried out by means of an osmotic machine. The machine consists, in its original condition, of a cylinder provided with a semipermeable piston, in the bottom of which, below the piston, is one molecule of the solid substance, while above is that amount of the pure solvent in which the solid substance is soluble. The solid substance is moistened with an infinitely small amount of the saturated solution, and the osmotic pressure on the piston is just balanced by a corresponding weight. The machine is placed in a very large heat reservoir at $T^{\circ}$, and the following cyclical process consisting of five parts is carried out :

Part 1 . By an indefinitely small decrease of the weight on the piston the latter is allowed to rise until the solid substance is dissolved. Let the concentration of the resulting saturated solution be expressed by $s$.

Part II. The piston is fastened and the machine placed in a very large heat reservoir at $(T+d T)^{\circ}$.

Part III. The piston is set free after loading it with a weight equal to the osmotic pressure; the weight is then increased by an indefinitely small amount, and the piston allowed to sink until the concentration of the solution has risen from $s$ to that of the solution saturated at $T+d T$; that is, $s+d s$.

Part $I V$. The piston is now allowed to sink still further, until the molecule of the substance has separated out of the solution.

1 Ztschr, phys. Chem., 17, 547. 
Part $V$. Finally the machine is placed in the heat reservoir at $T^{\circ}$.

The cyclical process is now completed and the original condition restored. The Second Law of Energetics gives now the relation which must exist between the work $d A$ performed on the system and the amount of heat $Q$ which is transferred from one temperature to the other. This relation is

$$
d A=Q \frac{d T}{T} .
$$

We will next determine the amount of work performed in the above-described process. The work done in each part consists in a change of volume taking place at a constant pressure, and is therefore equal to the product of these two quantities. According to the Avogadro-van't Hoff principle, the osmotic pressure is equal to $n R T$, where $n$ denotes the number of molecules present. As above, the concentration of the solution saturated at $T^{\circ}$ is $s$, and at $T+d T^{\circ}$ is $s+d s$. Further, let the number of molectles which is present in the solution of one molecule of the solid substance at $T^{\circ}$ be $i$, and at $T+d T^{\circ}$ be $i+d i$. Then the following values of the change in volume $(\Delta v)$, of the pressure $(P)$, and of the work performed $(A)$, in the separate parts of the process, are:

Part I. $(\Delta v)_{\mathrm{i}}=\frac{\mathrm{I}}{\mathrm{s}} ; P_{\mathrm{i}}=$ is $R T ; A_{\mathrm{i}}=-i R T$.

Part II. $(\Delta v)_{\mathrm{ii}} \mathrm{o} ;=A_{\mathrm{ii}}=0$.

Part III. $(\Delta v)_{\mathrm{iii}}=-\frac{I}{s}+\frac{I}{s+d s} ; \quad P_{\mathrm{jii}}=i s R(T+d T)$;

$$
A_{i i \mathrm{i}}=i R T d \log s \text {. }
$$

Part IV. $(\Delta v)_{\mathrm{iv}}=-\frac{\mathrm{I}}{s+d s} ; P_{\mathrm{iv}}=$

$$
(i+d i)(s+d s) R(T+d T) ;
$$

$$
A_{\mathrm{iv}}=i R T+i R d T+R T d i \text {. }
$$

Part V. $(\Delta v)_{v}=0 ; A_{v}=0$.

The total work done in the process is, therefore,

$$
d A=i R(T d \log i s+d T) .
$$


On the other hand, the amount of heat, $Q$, absorbed at the temperature $T$, is evidently the sum of the heat of solution $L$ in the ordinary sense (without work being done) and the external work; that is to say, $Q=L+i R T$. If these values of $d A$ and $Q$ are substituted in equation (6), the expression of the Second Law, we obtain

$$
\frac{L}{R T^{2}}=\frac{i d \log i s}{d T}
$$

which is the original van't Hoff equation. Attention may be especially called to the fact that in this cyclical process no opportunity is offered for the introduction of van Laar's correction.

This equation is evidently independent of any dilution-law whatever, and of the number of ions into which the electrolyte is dissociated. If the electrolyte breaks up to an extent $a$ into $n$ ions, $i=\mathrm{I}+(n-\mathrm{I}) \boldsymbol{a}$, whence follows :

$$
\frac{L}{R T^{2}}=[\mathrm{I}+(n-\mathrm{I}) a] \frac{d \log s}{d T}+(n-\mathrm{I}) \frac{d a}{d T} \text {. }
$$

Now if the electrolyte follows the dilution-law

$$
K=\frac{(a s)^{2}}{(\mathrm{I}-a) s}
$$

where $v$ may have any value, then we obtain, by differentiation,

$$
\frac{d a}{d T}=\frac{a(\mathrm{I}-a)}{v-(\nu-\mathrm{I}) a} \frac{d \log K}{d T}-\frac{(v-\mathrm{I}) a(\mathrm{I}-a)}{v-(\nu-\mathrm{I}) a} \frac{d \log s}{d T} .
$$

By substitution of this value in equation (6) we obtain

$$
\frac{L}{R T^{2}}=\frac{v+(n-v) a}{v-(v-\mathrm{I}) a} \frac{d \log s}{d T}+\frac{(n-\mathrm{I}) a(\mathrm{I}-a) d \log K}{v-(\nu-\mathrm{I}) a} \ldots(7)
$$

This equation is rigidly exact and general ; in it it is presupposed only that the electrolyte follows some exponential dilutionlaw of the assumed form.

In the two limiting cases where $a=\mathrm{I}$, and $a=0$, the equation becomes simplified to

$$
\frac{L}{R T^{2}}=\frac{n d \log s}{d T}, \text { and } \frac{L}{R T^{2}}=\frac{d \log s}{d T},
$$

as the theory requires. In general, however, in the calculation of the heat of solution, a knowledge not only of the temperature- 
change of the solubility, but also of that of the dissociation-constant, is necessary. The latter change has in the case of most electrolytes been shown to be so small that for practical purposes the last member may ordinarily be neglected without a large error, a simplification which is permissible especially in the case of very weakly and very strongly dissociated electrolytes, for the coefficients of $d \log K: d T$ disappear when $a$ approaches zero or unity.

We will now consider two special cases : first, where the electrolyte is di-ionic and follows the theoretical (Ostwald) dilutionlaw, where it is, for example, a weak acid or base ; and second, where the electrolyte is di-ionic and follows the empirical (van't Hoff) dilution-law, where it is, for example, a salt. In both cases $n=2$; in the first case $v=2$, and in the second $v=\frac{3}{2}$. By substituting these values in equation ( 7 ) and omitting the last member we obtain

$$
\begin{aligned}
\frac{L}{R T^{2}} & =\frac{2}{2-a} \frac{d \log s}{d T} \text { when } v=2, \ldots \ldots(8) \\
\text { and } \frac{L}{R T^{2}} & =\frac{3+a}{3-a} \frac{d \log s}{d T} \text { when } v=\frac{3}{2} \ldots \ldots(9)
\end{aligned}
$$

The first formula is identical with that obtained by van Laar; the second is, however, essentially different from his, which reads

$$
\frac{L}{R T^{2}}=\frac{3}{3-a} \frac{d \log s}{d T} \ldots \ldots \ldots \ldots \text { (10) }
$$

\section{COMPARISON OF THE CALCULATED AND THE OBSERVED HEATS OF SOLUTION.}

In the following tables the results of the calculation of the heats of solution according to the three formulas (8), (9), and (IO), given above, are placed together beside the experimentally found values for the four substances used by van Laar. Only those values in the next to the last column are calculated by me $;{ }^{1}$ the rest are those of van Laar.

1 These values were obtained from those of van Laar in the preceding column by multiplication by $\frac{3+a}{3}$. The values of $a$ used are those derived by van Laar from conductivity measurements; namely, for silver acetate, 0.713 ; for silver propionate 0.744 ; for silver isobutyrate, 0.718 ; and for 0 -nitrobenzoic acid, 0.303 . 
HEATS OF SOLUTIOX.

Silver acetate ........ 4369

\section{Ostwald's \\ dilution-1aw \\ assumed.}

Silver propionate...$\cdots 3789$

Silver isobutyrate...... 2715

0 -Nitrobenzoic acid..... 7167

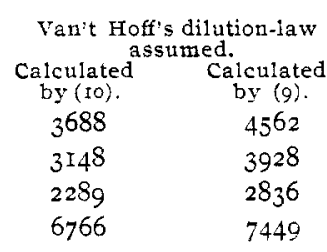

Experi-

menta1!y

foutd.

4613

3980

2860

7083

From the fact that the values in the first column of numbers, which were obtained with the help of the Ostwald law, agree much more closely with the experimental values than do those of the second column, which were calculated by means of van't Hoff's dilution-law and the equation of van' 't Hoff as corrected by van Laar, van Laar concluded that salts in reality follow Ostwald's dilution-law, and not van 't Hoff's, as is to be inferred from the conductivity; in other words, the conductivity gives, according to him, incorrect values of the dissociation. Further support of this latter assumption he finds in the fact that in the case of the three salts, even the values calculated with the help of Ostwald's law differ from the experimental ones by several per cent. But I have proved above, as I believe, that the equation of van Laar, in the deduction of which van't Hoff's dilutionlaw was presupposed, is incorrect, and that the true expression is equation (9). If one is to decide between the two dilutionlaws, the values of the first and third columns of numbers must, therefore, be compared with those of the fourth. This comparison shows now just the opposite result; in the case of the three salts, the values deduced from van 't Hoff's law agree almost completely (within 0.8 or I.3 per cent.) with the actual ones, while those from Ostwald's law deviate by about five per cent. Moreover, it is especially worthy of note that the nitrobenzoic acid, which, like all weak acids, follows Ostwald's law, possesses a heat of solution which is in accord with the assumption of the validity of this law, but which is not consistent with van't Hoff's law. The results are therefore in perfect agreement with the usual assumptions based on conductivity in regard to the dissociation of the substances in question, and not only is the argument of van Laar refuted, but at the same time a new proof is furnished of the validity of van't Hoff's dilution-law in the 
case of salts, and of the reliability of the dissociation-values determined from conductivity measurements.

4. ELECTRICAL CONDUCTIVITY AND INVERSION-VELOCITY IN MIXTURES OF WATER AND ALCOHOL.

Van Laar finds a further proof of his view in the fact that the rate of inversion of sugar by hydrochloric acid, in mixtures of alcohol and water, is essentially different from that in pure water, although according to Cohen ${ }^{1}$ the degree of dissociation of the acid, as determined by its conductivity in the two solvents, is the same. Cohen ${ }^{2}$ also considers this fact as leading to the conclusion that "in solutions in mixtures of water and alcohol the electrical conductivity is not an entirely correct measure of the degree of dissociation."

It is remarkable that this conclusion has been drawn by two separate investigators; for it appears to me obviously unjustified. Even if the concentrations of the hydrogen ions are the same, it is by no means to be expected that the rates of inversion in the different solvents would be equal. For, aside from the fact that the catalytic activity of the hydrogen ions is not necessarily constant, it is clear that the concentration of one of the reacting substances, the water, undergoes a change; and it would be indeed remarkable if this change had no influence on the rate of inversion. It is not improbable that the ions of the water play an important part in the hydrolysis of the sugar, and it has been shown by Löwenherz ${ }^{3}$ that the addition of alcohol considerably reduces the dissociation of water. It is therefore entirely inadmissible to ascribe to the inversion results such a significance as van Laar and Cohen do.

5. VAN LAAR'S "EXPLANATION OF THE DEVIATIONS FROM OSTWALD'S LAW."

It seems superfluous to discuss the hypothesis of van Laar on the cause of the incorrectness of the dissociation-values derived from the conductivity, since, from what has been said above, no reason exists to doubt their correctness. A remark in regard

1 Zischr, phys. Chem., 25, 4t.

2 Ibid., p. 44.

3 Ibid, 20, 294. 
to it may be, however, not without interest. If I correctly understand his hypothesis, its essential feature is that the Joule heat-effect which is continuously produced by the current during the measurement of the conductivity, causes a localized increase of temperature around the ions, as a result of which the dissociation is temporarily changed. Against this hypothesis, however, there exists the following fatal objection : if the dissociation and conductivity are affected by the heat produced by the current, then the observed values of these would depend on the strength of the current employed, which is known not to be the case.

6. EXISTING DETERMINATIONS OF THE DISSOCIATION BY INDEPENDENT UETHODS.

In his article, van Laar pays no attention to previously published experiments which bear upon the reliability of the contductivity method of determining dissociation; yet certain investigations have been carried out, the purpose of which was to answer this very question. In the first place may be considered the recent investigations of Jones, ${ }^{1}$ Loomis, ${ }^{2}$ Abegg, ${ }^{3}$ and Raoult, ${ }^{4}$ on the lowering of the freezing-point of water by cerium salts. Of these the two salts, potassium chloride and sodium chloride, have been most carefully studied, for the lowerings of freezing-point caused by them have been determined by all four of the mentioned investigators.

In order to show now the extent of the agreement of the values with one another and with the results of the conductivity method, I have brought together in the following table the calculated dissociation-values for the concentrations 0.01 to 0.1 normal. In the calculations from the freezing-point lowerings I. $85^{\circ}$ was assumed as the molecular lowering of water. ${ }^{5}$ The conductivity determinations of Kohlrausch were made use of in calculating the dissociation-values by this method.

\footnotetext{
1 Ztschr. phys. Chem., II, I10, 529; 12, 623.

2 Wied. Ann., 51, 500; 57, 495.

8 Zischr. phys, Chem., 20, 207.

4 Compt. rend., 124, $885 ; 125,75 \mathrm{I}$.

5 Compare Abegg : Wied. Ann., 64, 499.
} 
Dissociation-values.

Potassium chloride.

Sodium chloride.

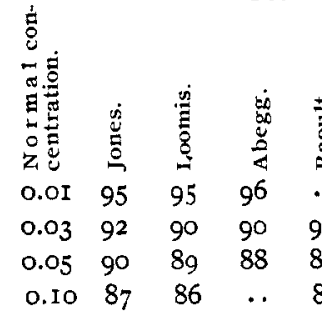

\begin{tabular}{|c|c|c|c|c|c|c|c|}
\hline 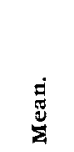 & 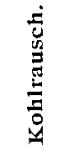 & $\stackrel{\dot{\theta}}{\stackrel{g}{2}}$ & 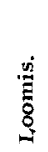 & 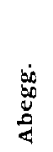 & $\begin{array}{l}\dot{\Xi} \\
\vec{Z} \\
\stackrel{\Xi}{Z}\end{array}$ & 离 & 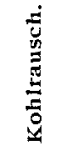 \\
\hline 95.3 & 94.4 & 95 & 99 & IO5 & $\cdot$ & 99.7 & 93.7 \\
\hline 90.8 & 9r.I & 92 & 93 & 92 & 97 & 93.5 & 89.6 \\
\hline 88.8 & 89.1 & 90 & $9 \mathrm{I}$ & 92 & 93 & $9 \mathrm{I} .5$ & 87.3 \\
\hline 86.0 & 86.2 & 88 & 88 & 88 & 89 & 88.2 & 84.2 \\
\hline
\end{tabular}

It is seen that, in the case of potassium chloride, the four separate values by the freezing-point method agree well with one another, and the averages of them are almost identical with the dissociation-values calculated from Kohlrausch's conductivity measurements. In the case of sodium chloride, however, the agreement between the freezing-point results of the separate observers is not so complete, and their averages are about four per cent. higher than the values to which the conductivity leads. Whether this latter result arises from a constant error in the freezing-point determinations or from a theoretical inaccuracy in one of the two methods, can not, of course, be determined at present. In view of this fact it must be admitted, to be sure, that these freezing-point measurements do not contribute much towards answering the question regarding the reliability of the conductivity method. Nevertheless a certain significance is to be attributed to the close agreement in the case of the potassium chloride, the best investigated salt.

In another way, however, more decisive evidence of the reliability of that method has been furnished. From the phenomenon of solubility effect it is possible to determine dissociation-values, and experiments of Noyes and Abbot ${ }^{1}$ have shown that the values so determined in the case of certain thallium salts are in accordance with those derived from the conductivity. For details reference is made to the original article; but the final results may be presented here. The first two columns of figures in the following table show the dissociation-values which were calculated from independent solubility experiments with two pairs of salts. The last column contains the corresponding dissociation-values, which were derived from the conductivity.

1 Ztschr. phys. Chem., I6, 136 . 


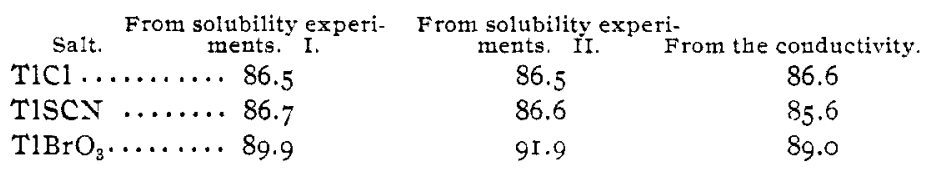

These entirely distinct methods, freezing-point-lowering and solubility-effect, lead therefore to nearly the same dissociationvalues. That the electrical conductivity furnishes essentially correct dissociation-values in the case of di-ionic salts in moderately dilute solution, is therefore probable. At any rate, more weighty reasons than those brought forward by van Laar must be discovered before this method should be discredited.

\section{THE ERROR IN CARBON DETERMINATIONS MADE WITH THE USE OF WEIGHED POTASH BULBS.}

BI GeORGE AUCH:

Recelved June 2, 1898 .

TH

HE difficulty, or impossibility even, of obtaining good carbon results in damp weather has been so often spoken of that anything further on the same subject would seem superfluous. Nevertheless the writer ventures a few remarks. In determining carbon by the dry combustion method it was found that, after the prolong had been in use a few times, a loss of moisture occurred as shown by the gain in weight of a potash bulb containing strong sulphuric acid, placed after the prolong. But it was later discovered that this loss was mainly due to the fact of the copper oxide in the preheating furnace being too fine and packed in too tightly. With coarser copper oxide, and a consequently easier passage of gas and air, and therefore less force and speed in aspiration, the amount of moisture lost from the prolong was much lessened, and the increase in the loss very steady and gradual so that a determination of it by means of a sulphuric acid bulb ${ }^{1}$ would only be necessary once in a while, and might be altogether dispensed with by a frequent change in the calcium chloride of the prolong. This agrees with Dr. Drown's experience who found by many trials that the prolong retained all the moisture passing into it from the potash bulbs; but, while still using the sulphuric acid bulb, some gains in its

I or better, a snall calcium-chioride tube, as it exposes less surface for the con. densation of moisture on a damp day. 